\title{
Clinical Utility and Impact of the Metagenomic Microbial Plasma Cell-Free DNA Next-Generation Sequencing Assay on Treatment Decision: A Single- Center Retrospective Study
}

\author{
Akira Shishido ( $\sim$ ashishido@som.umaryland.edu ) \\ University of Maryland Medical Center \\ Myint Noe \\ Lee Health \\ Kapil Saharia \\ University of Maryland School of Medicine \\ Paul Luethy \\ University of Maryland School of Medicine
}

\section{Research Article}

Keywords: cell-free DNA, transplant infectious disease, next-generation sequencing, Karius @ , sepsis

Posted Date: January 31st, 2022

DOI: https://doi.org/10.21203/rs.3.rs-1160543/v1

License: (c) (i) This work is licensed under a Creative Commons Attribution 4.0 International License. Read Full License

Version of Record: A version of this preprint was published at BMC Infectious Diseases on April 13th, 2022. See the published version at https://doi.org/10.1186/s12879-022-07357-8. 


\section{Abstract \\ Background}

Metagenomic next-generation sequencing (mNGS) of microbial cell-free DNA (mcfDNA) allows for noninvasive pathogen detection from plasma. However, there is little data describing the optimal role for this assay in real-world clinical decision making.

\section{Methods}

We performed a single-center retrospective cohort study of adult patients for whom a mcfDNA test was sent between May 2019 and February 2021. Clinical impact was arbitrated after review and discussion of each case.

\section{Results}

A total of 80 patients were included. The most common reason for sending the assay was unknown microbiologic diagnosis ( $78 \%)$, followed by avoiding invasive procedures $(14 \%)$. The test had a positive impact in $34(43 \%)$, a negative impact in $2(3 \%)$, and uncertain or no impact in $44(55 \%)$. A positive impact was observed in solid organ transplant recipients (SOTR, $71.4 \%, \mathrm{p}=0.003$ ), sepsis $(71.4 \%$, $p=0.003$ ), and those receiving antimicrobial agents for less than 7 days prior to mcfDNA testing (i.e., $61.8 \%, p=0.004)$.

\section{Conclusion}

Clinical utility of mcfDNA testing was highest in SOTR and patients with sepsis. Prolonged antimicrobial use prior to mcfDNA testing limited the utility of the assay. Prospective studies evaluating the utility of mcfDNA assays should be performed to further clarify its role in clinical management.

\section{Key Points}

This is a retrospective study evaluating the clinical utility of mcfDNA testing at a single center. mcfDNA positively impacted clinical care in $43 \%$ of cases. Patients admitted with sepsis, patients receiving antibiotics for less than 7 days, and solid organ transplant recipients derived the most benefit from mcfDNA testing.

\section{Introduction}

Clinical metagenomic next-generation sequencing (mNGS) is an emerging diagnostic modality that comprehensively analyzes all genetic material in a given sample of fluid or tissue. ${ }^{1}$ mNGS platforms 
sequence millions of small DNA and/or RNA fragments in parallel. ${ }^{1}$ Bioinformatic analyses then match sequences to reference genomes for identification. ${ }^{1}$ While most conventional molecular diagnostic assays target a single or limited number of pathogens, mNGS of microbial cell-free DNA (mcfDNA) allows for broad-range pathogen detection. ${ }^{1,2}$ The Karius@ test (Karius, Redwood City, California) emerged in 2016 making mNGS of mcfDNA widely available. The assay amplifies small fragments of mcfDNA and then matches the sequences to a bank of reference genomes that can reportedly identify 1250 bacteria, viruses, fungi and parasites. ${ }^{2}$

Understandably, a non-invasive assay able to detect multiple pathogens is an attractive prospect for clinicians. However, in comparison to conventional diagnostics, mcfDNA and mNGS are expensive and do not provide antimicrobial susceptibility results. Current data to define the optimal clinical context for the use of the mcfDNA assay is limited. The use of mcfDNA at our institution has been restricted to tertiary testing in scenarios where conventional assays do not provide a diagnosis or situations that require morbid invasive procedures. mcfDNA sequencing has been studied as a complimentary assay in the rapid diagnosis of sepsis, culture-negative endocarditis, pneumonia, invasive fungal infections, brain abscesses and more recently as an adjunct to conventional microbiology cultures in bloodstream infections and prosthetic joint infections. ${ }^{2-8}$ Despite results of recent clinical trials and case reports demonstrating the potential value of mcfDNA as a diagnostic tool, the overall impact of mcfDNA testing on clinical care is less certain. For example, a retrospective cohort study by Hogan and colleagues evaluating the clinical impact of mcfDNA found that despite a positivity rate of $61 \%$, mcfDNA had a positive clinical impact in only $7.3 \%$ of cases. ${ }^{9}$ This is in contrast to a study by Rossoff et al. in which $56 \%$ of samples sent for mcfDNA provided clinically relevant information. ${ }^{10}$ Given the clinical equipoise, we performed a single center, retrospective study to assess the clinical impact of mcfDNA testing stratified by patient comorbidities, clinical syndromes, days of antimicrobial therapy and indication for testing to identify a context, if any, in which the assay may have the highest clinical utility.

\section{Methods}

\section{Ethical Considerations}

The study protocol was approved by the institutional review board (IRB) at University of Maryland, Baltimore (UMB).

\section{Study Design}

We performed a retrospective cohort study of adult patients for whom a mcfDNA test was sent from our institution between May 2019 and February 2021. We predefined clinical impact categories (Table 1) based on criteria used by Hogan et al. and performed comprehensive record reviews for each case. We included and stratified data by patient co morbidities, infectious syndromes, duration of antimicrobial therapy prior to mcfDNA testing, reasons for sending the test, and final clinical diagnosis. Cases that fit within multiple predefined categories were included and analyzed in all categories to which they applied. 
Clinical impact was arbitrated by all authors based on the actions of the treatment team and patient outcome after review and discussion of each case. The mcfDNA assay was performed by Karius $\odot$ as described by Blauwkamp et al. 2019. ${ }^{2}$

Table 1

Clinical Impact Categories and their Predefined Criteria

\begin{tabular}{ll}
\hline Category & Definition \\
\hline Positive & Test result led to a new diagnosis when conventional tests were negative. \\
\hline Test result confirmed clinical diagnosis. \\
\hline Test result led to an earlier diagnosis. \\
\hline Test result negated invasive or costly procedures or tests. \\
\hline Test result helped reduce length of hospital stay. \\
\hline Test result led to the initiation of appropriate antimicrobial therapy. \\
\hline Test result led to de-escalation or discontinuation of antimicrobial therapies. \\
\hline Negative & Test result led to unnecessary antimicrobial treatment. \\
\hline Test result led to unnecessary diagnostic investigation or procedures. \\
\hline $\begin{array}{l}\text { Test result led to an unnecessarily prolonged hospital stay. } \\
\text { impact }\end{array}$ & $\begin{array}{l}\text { Test result did not change any clinical management or unable to determine the } \\
\text { clinical impact. }\end{array}$
\end{tabular}

Legend: Test Result - result of microbial cell-free DNA assay; conventional tests - standard serological, microbiological, and molecular, histopathological and biochemical results.

\section{Statistical Analysis}

Categorical variables were summarized using frequency and percentages and continuous variables were summarized using median and quartiles as the variables were non-normal. Comparative analysis of categorical variables was conducted by the Fisher's exact test or chi-square test as appropriate and continuous data were compared using Mann-Whitney U test. Statistical tests were performed using SPSS (IBM SPSS Statistics for Windows, Version 27.0. Armonk, NY: IBM Corp) with p-values $\leq 0.05$ as the significance threshold.

\section{Results}

A total of 80 patients had mcfDNA testing over the course of the study period (Table 2). The median age was 54.5 and $60 \%$ of the patients were male. The most common reason for sending the assay was unknown microbiologic diagnosis (78\%), followed by avoiding invasive procedures $(14 \%)$, confirmatory 
testing (5\%) and early diagnosis (3\%). Forty-five patients (56\%) were immunocompromised (Table 3$)$. The most common immunocompromising condition was hematologic malignancy (27\%), followed by solid organ transplantation $(26 \%)$. Fourteen patients $(18 \%)$ had prosthetic hardware or grafts, the majority of which were prosthetic heart valves $(10 \%)$ followed by vascular grafts $(7 \%)$. The most common clinical syndrome was respiratory failure/pneumonia (31\%) followed by sepsis/septic shock (15\%). Thirty-seven patients $(51 \%)$ received more than 7 days of antimicrobial therapy prior to mcfDNA testing.

Table 2

Patient Demographic and Baseline Characteristics

\begin{tabular}{|c|c|}
\hline Total patients & $\mathrm{N}=80$ \\
\hline Age (years), median (+/-SD) & $54.5(15.59)$ \\
\hline \multicolumn{2}{|l|}{ Gender, n (\%) } \\
\hline Female & $32(40)$ \\
\hline Male & $48(60)$ \\
\hline \multicolumn{2}{|l|}{ Reason for mcfDNA Assay, n (\%) } \\
\hline Microbiologic diagnosis unknown & $74(78)$ \\
\hline Avoid invasive diagnostic procedure & $13(14)$ \\
\hline Confirmatory test & $5(5)$ \\
\hline Early diagnosis & $3(3)$ \\
\hline \multicolumn{2}{|l|}{ Types of Clinical Impact, n (\%) } \\
\hline Negative & $2(3)$ \\
\hline Positive & $34(43)$ \\
\hline Uncertain or No impact & $44(55)$ \\
\hline \multicolumn{2}{|c|}{ Consistency with Final Clinical Diagnosis, n (\%) } \\
\hline Yes & $52(65)$ \\
\hline No & $25(31)$ \\
\hline NA & $3(4)$ \\
\hline
\end{tabular}


Table 3

Patient Characteristics and Relationship to Clinical Impact †

\begin{tabular}{|c|c|c|c|c|}
\hline Comorbidities & $\begin{array}{l}\mathrm{n} \\
(\%)\end{array}$ & $\begin{array}{l}\text { Uncertain or No } \\
\text { impact, } \mathrm{n}(\%)\end{array}$ & $\begin{array}{l}\text { Positive } \\
\text { Impact, } \mathrm{n}(\%)\end{array}$ & $\begin{array}{l}\mathrm{P} \text { - } \\
\text { value }\end{array}$ \\
\hline Immunocompromised & $\begin{array}{l}45 \\
(56)\end{array}$ & $24(53.3)$ & $21(46.7)$ & 0.522 \\
\hline Organ transplant & $\begin{array}{l}21 \\
(26)\end{array}$ & $6(28.6)$ & 15 (71.4) & 0.003 \\
\hline Stem cell transplant & $\begin{array}{l}3 \\
(4)\end{array}$ & $3(100)$ & 0 & \\
\hline Solid tumor & $\begin{array}{l}2 \\
(2)\end{array}$ & $2(100)$ & 0 & \\
\hline Hematologic malignancy & $\begin{array}{l}22 \\
(27)\end{array}$ & $15(68.2)$ & $7(31.8)$ & 0.189 \\
\hline HIV/AIDS & $\begin{array}{l}1 \\
(1)\end{array}$ & $1(100)$ & 0 & \\
\hline Autoimmune disease & $\begin{array}{l}3 \\
(4)\end{array}$ & $1(33.3)$ & $2(66.7)$ & \\
\hline Hardware or prosthesis & $\begin{array}{l}14 \\
(18)\end{array}$ & $7(50)$ & $7(50)$ & 0.593 \\
\hline Vascular graft & $\begin{array}{l}6 \\
(7)\end{array}$ & $4(66.7)$ & $2(33.3)$ & 0.691 \\
\hline Prosthetic joint or orthopedic hardware & $\begin{array}{l}1 \\
(1)\end{array}$ & 0 & $1(100)$ & \\
\hline Mechanical Cardiac Device & $\begin{array}{l}1 \\
(1)\end{array}$ & 0 & $1(100)$ & \\
\hline Prosthetic valve & $\begin{array}{l}8 \\
(10)\end{array}$ & $3(37.5)$ & $5(62.5)$ & 0.285 \\
\hline Diabetes & $\begin{array}{l}13 \\
(16)\end{array}$ & $6(50)$ & $6(50)$ & 0.626 \\
\hline \multicolumn{5}{|l|}{ Infectious Syndrome/Clinical Diagnosis } \\
\hline Sepsis/Septic shock & $\begin{array}{l}14 \\
(15)\end{array}$ & $4(28.6)$ & $10(71.4)$ & 0.02 \\
\hline Bacteremia & $\begin{array}{l}3 \\
\text { (3) }\end{array}$ & $1(33.3)$ & $2(66.7)$ & \\
\hline Vascular graft infection & $\begin{array}{l}7 \\
\text { (7) }\end{array}$ & $4(57.1)$ & $3(42.9)$ & 1 \\
\hline Endocarditis & $\begin{array}{l}13 \\
(13)\end{array}$ & $6(46.2)$ & $7(53.8)$ & 0.414 \\
\hline
\end{tabular}




\begin{tabular}{|c|c|c|c|c|}
\hline Respiratory failure/pneumonia & $\begin{array}{l}30 \\
(31)\end{array}$ & $14(48.3)$ & $15(51.7)$ & 0.265 \\
\hline Bone/Joint infection & $\begin{array}{l}4 \\
(4)\end{array}$ & $3(75)$ & $1(25)$ & \\
\hline CNS infection (meningoencephalitis) & $\begin{array}{l}10 \\
(10)\end{array}$ & $7(77.8)$ & $2(22.2)$ & 0.285 \\
\hline Fever unknown origin & $\begin{array}{l}10 \\
(10)\end{array}$ & $5(50)$ & $5(50)$ & 0.74 \\
\hline Unexplained leukocytosis & $\begin{array}{l}2 \\
(2)\end{array}$ & $1(50)$ & $1(50)$ & \\
\hline Sinusitis & $\begin{array}{l}1 \\
(1)\end{array}$ & $1(100)$ & 0 & \\
\hline Skin and soft tissue infection & $\begin{array}{l}1 \\
(1)\end{array}$ & 0 & $1(100)$ & \\
\hline Others & $\begin{array}{l}1 \\
(1)\end{array}$ & $1(100)$ & 0 & \\
\hline $\begin{array}{l}\text { Antimicrobial agents administered prior to } \\
\text { mcfDNA test }\end{array}$ & $\begin{array}{l}72 \\
(90)\end{array}$ & $39(55.7)$ & $31(44.3)$ & 1 \\
\hline Less than 7 days & $\begin{array}{l}35 \\
(49)\end{array}$ & $13(38.2)$ & $21(61.8)$ & 0.004 \\
\hline More than 7 days & $\begin{array}{l}37 \\
(51)\end{array}$ & $26(72.2)$ & $10(27.8)$ & 0.004 \\
\hline No antimicrobial agents prior to mcfDNA test & $\begin{array}{l}8 \\
(10)\end{array}$ & $5(62.5)$ & $3(37.5)$ & 1 \\
\hline \multicolumn{5}{|l|}{ Final Diagnosis } \\
\hline Bacterial & $\begin{array}{l}26 \\
(31)\end{array}$ & $15(60)$ & $10(40)$ & 0.661 \\
\hline Fungal & $\begin{array}{l}21 \\
(25)\end{array}$ & $12(57.1)$ & $9(42.9)$ & 0.937 \\
\hline Viral & $\begin{array}{l}10 \\
(12)\end{array}$ & $6(60)$ & $4(40)$ & 1 \\
\hline Non-infectious & $\begin{array}{l}28 \\
(33)\end{array}$ & $14(51.9)$ & $13(48.1)$ & 0.555 \\
\hline $\begin{array}{l}\text { Days of Hospitalization before sending } \\
\text { mcfDNA test, Median (Q1, Q3) }\end{array}$ & & $11.00(5.00,21.50)$ & $\begin{array}{l}9.50 \\
(3.00,18.25)\end{array}$ & 0.361 \\
\hline \multicolumn{5}{|c|}{$\begin{array}{l}\text { Legend: HIV/AIDS: Human immunodeficiency virus/Acquired immunodeficiency syndrome; CNS: } \\
\text { Central Nervous System. }\end{array}$} \\
\hline
\end{tabular}


The mcfDNA test result was consistent with the final diagnosis in $65 \%$ of cases and had a positive impact in 34 cases (43\%), a negative impact in 2 cases (3\%), and uncertain or no impact in 44 cases (55\%) (Table 2). The only patient characteristic associated with a positive impact from mcfDNA testing was solid organ transplantation $(71.4 \%, p=0.003)$. Other variables associated with a positive impact from mcfDNA testing in univariate analysis were presence of sepsis $(71.4 \%, p=0.02)$ and antibiotic duration less than 7 days prior to mcfDNA testing $(61.8 \%, p=0.004)$. No other patient comorbidities, clinical syndromes or variables analyzed yielded a statistically significant association with positive impact from mcfDNA testing.

Pathogens were identified via mcfDNA test in $49 / 80$ cases. Of the pathogens identified $31 \%$ were bacteria, $10 \%$ were viruses, and $21 \%$ were mold/fungi (Table 3 ). In cases in which pathogens were identified, $55.1 \%$ yielded a positive impact (Table 4 ). In 31/80 cases, mcfDNA testing yielded a negative result. In 7 cases, the negative test supported a non-infectious etiology of the patient's syndrome and antibiotics were either de-escalated or stopped. Positive impact was driven primarily by mcfDNA test results leading to de-escalation (47\%) of antimicrobial therapy (Table 5).

Table 4

mcfDNA result type and Relationship to Clinical Impact

\begin{tabular}{|lll|}
\hline Result Type & $\begin{array}{l}\text { Uncertain, No Impact or Negative Impact, } \mathrm{n} \\
(\%)\end{array}$ & $\begin{array}{l}\text { Positive Impact, } \mathrm{n} \\
(\%)\end{array}$ \\
\hline $\begin{array}{l}\text { Positive with quantitative } \\
\text { result }\end{array}$ & $19(45.2 \%)$ & $23(54.8 \%)$ \\
\hline Positive with qualitative result & $3(42.9 \%)$ & $4(57.1 \%)$ \\
\hline Negative & $24(77.4 \%)$ & $7(22.6 \%)$ \\
\hline $\begin{array}{l}\text { Did not Meet QC and was not } \\
\text { run }\end{array}$ & 3 & 0 \\
\hline Legend: QC - Quality Control, as specified by Karius@ & \\
\hline
\end{tabular}


Table 5

mcfDNA assay with positive impact, reason for positive impact

\begin{tabular}{|ll|}
\hline Reason for positive Impact & $\mathbf{N}(\%)$ \\
\hline Led to new diagnosis & $6(17.6)$ \\
\hline Led to confirmation of diagnosis & $11(32.4)$ \\
\hline Led to earlier diagnosis & $2(5.9)$ \\
\hline Avoided invasive procedure & $3(8.8)$ \\
\hline Led to appropriate antimicrobials & $6(17.6)$ \\
\hline Led to de-escalation of antimicrobials & $16(47.1)$ \\
\hline Legend: N/A & \\
\hline
\end{tabular}

\section{Discussion}

While mNGS is an attractive diagnostic modality for its non-invasive broad-range pathogen detection, its clinical utility and impact on clinical decision-making remains poorly defined. In this single-center retrospective cohort study, we show that the overall clinical utility of mNGS for pathogen identification remains low (43\%). Additionally, we identified three contextual factors within our cohort wherein a mNGS testing had a positive impact on clinical decision-making: SOTR $(71.4 \%, p=0.003)$, sepsis $(71.4 \%, p=0.02)$ and antimicrobial therapy for fewer than 7 days prior to assay collection $(61.8 \%, p=0.004)$. The clinical impact was driven primarily by mcfDNA testing leading to de-escalation of antimicrobials and confirming clinical diagnosis (Table 5).

Our finding of $43 \%$ overall clinical benefit is higher than prior studies evaluating clinical impact of mNGS for pathogen detection in CSF (3.4\%) and plasma (7.3\%). ${ }^{9,11}$ This may be due in part because mcfDNA testing is strictly regulated within our institution. All mcfDNA testing is reviewed by an Infectious Disease specialist in consultation with the clinical microbiology director. Therefore, all mcfDNA testing is performed on a narrow, vetted patient population. Additionally, 33\% of patients in the study by Hogan et al. had a preestablished microbiological diagnosis through conventional testing, whereas in our study mcfDNA testing was often performed when diagnosis was in question. ${ }^{9}$ Lack of clinical impact was most commonly due to identification of a new organism that was not acted upon or confirmation of a conventional result that was not acted upon. ${ }^{9}$ In our analysis, we designated mcfDNA confirmation of diagnosis that led to de-escalation of antimicrobials as a positive impact and found this to be the primary driver of clinical benefit. Additionally, our results appear consistent with a similarly executed study by Rossoff et. al that explored the diagnostic capabilities of mcfDNA testing for pediatric infections. ${ }^{10}$ While Rossoff et. al did not formally assess clinical outcomes on mcfDNA-based decision-making, they found 
that mcfDNA testing netted a higher yield of clinically relevant pathogens in immunocompromised patients $(61 \%)$ than in immunocompetent patients $(35 \%) .{ }^{10}$

The positive clinical impact observed in our study was largely driven by mcfDNA leading to de-escalation of antimicrobials. This benefit was often derived from situations in which the assay either identified pathogens that were felt to not be clinically relevant, or no pathogen at all (Table 5). While different from our study in its scope, a study by Eichenberger, et al. demonstrated that mcfDNA persisted in plasma well beyond conventional blood cultures in blood stream infections and that persistence was associated with an increased risk of metastatic infection. ${ }^{7}$ Taken together, the safe de-escalation or discontinuation of antimicrobials in the context of negative or decreasing levels of mcfDNA may highlight a role for mcfDNA in the realm of stewardship or determining antimicrobial course duration.

The fact that the clinical factors associated with positive clinical impact by mcfDNA testing were SOTR, sepsis and short antimicrobial courses may be due to both host and environmental factors. Immunocompromised patients who lack adequate T-cell responses may present with atypical presentations of infections that are less amenable to detection by conventional cultures. Additionally, these patients often receive broad empiric antimicrobial therapy thus reducing the yield of conventional cultures. Patients with sepsis may also have altered immune system function and receive broad therapy affecting conventional techniques.

That other immunosuppressed populations in our cohort (i.e., stem cell transplant patients) did not display the same level of benefit from mcfDNA testing may be explained by their underrepresentation and small contributing numbers to the overall data as well as standardized algorithmic approach to management. A recent study by Benamu et al. assessed the utility of early mcfDNA testing in patients with neutropenic fever by prospectively obtaining mcfDNA within 24 hours of fever onset. ${ }^{11}$ The authors concluded that mcfDNA testing could have allowed earlier optimization of antimicrobials in $47 \%$ of patients.13 While the utility of mcfDNA testing in this study was similar to our overall results, the lack of effect we observed specifically in hematologic malignancy and stem cell transplant patients compared to Benamu et al. may be due to several factors. First, real-world management of febrile neutropenia remains institution-specific and protocol-driven. Antimicrobial de-escalation may not occur even in the presence of identified pathogens. Therefore, the lack of observed utility in our study may be because the primary team did not change management based on the mcfDNA result even if it identified a true pathogen. Second, the prior study did not base utility on clinical outcomes, but instead on an arbitration of whether the mcfDNA result could have made an impact - thereby attenuating any potential algorithmic impact on clinical management results. Third, Benamu et al. collected mcfDNA within 24 hours of fever onset whereas patients in our study often had mcfDNA evaluated weeks into their course. Lastly, stem cell patients were underrepresented in our cohort.

The retrospective nature of the study comes with inherent limitations. Despite the basis of clinical impact of mNGS on the clinical team's management and patient outcome, the retrospective arbitration process to assign utility comes with inherent subjectivity. Additionally, the retrospective nature of the study does not 
allow for control of timing of testing and patient and disease characteristics, therefore there was considerable variability among factors and many underrepresented patient populations and diseases. Lastly, the small sample size and retrospective nature allow for only hypothesis-generating conclusions to be made.

In conclusion, while mNGS of mcfDNA is a promising technology for rapid microbial diagnosis, the exact clinical context and utility of the test remains undefined. Our study identifies several factors for which the assay may have a higher likelihood of providing clinical benefit: SOTR, sepsis and patients who have received fewer than 7 days of antimicrobial therapy. Positive clinical impact was driven primarily by deescalation of antimicrobial therapy suggesting a potential role for mdfDNA in the realm of stewardship. Further studies should explore this relationship and the utility of mcfDNA testing specifically in this context.

\section{Declarations}

\section{Ethics approval and consent to participate:}

This study protocol was approved by the Human Research Protections Office (HRPO) of the University of Maryland, Baltimore. This study was retrospective in nature and deemed exempt from informed consent by the HRPO. All research performed in this study was done in accordance with the standards set by the HRPO.

\section{Consent for publication:}

N/A

\section{Availability of data and materials:}

De-identified data are available as supplementary material (Supplementary Table 1) or by request to the corresponding author.

\section{Conflicts of Interest:}

The authors have no conflicts of interest to disclose.

\section{Funding:}

None

\section{Author Contribution:}

Original research performed by all authors.AS drafted original manuscript. MN, PL and KS contributed additional sections and references. All authors reviewed and approved the final manuscript.

\section{Acknowledgements:}


Nivya George (Institute of Human Virology, Baltimore, Maryland, USA) aided with statistical analysis. Preliminary analysis of this data was previously presented at IDWeek2021@ with abstract published in Open Forum Infectious Diseases. ${ }^{12}$

\section{References}

1. Chiu CY, Miller SA. Clinical metagenomics. Nature Reviews Genetics. 2019;20(6):341-355. doi:10.1038/s41576-019-0113-7

2. Blauwkamp TA, Thair S, Rosen MJ, et al. Analytical and clinical validation of a microbial cell-free DNA sequencing test for infectious disease. Nature Microbiology. 2019;4(4):663-674. doi:10.1038/s41564-018-0349-6

3. Hong DK, Blauwkamp TA, Kertesz M, Bercovici S, Truong C, Banaei N. Liquid biopsy for infectious diseases: sequencing of cell-free plasma to detect pathogen DNA in patients with invasive fungal disease. Diagnostic Microbiology and Infectious Disease. 2018;92(3):210-213. doi:10.1016/j.diagmicrobio.2018.06.009

4. Downey RD, Russo SM, Hauger SB et al. Identification of an Emergent Pathogen, Bartonella vinsonii, Using Next-Generation Sequencing in a Patient With Culture-Negative Endocarditis [published online ahead of print, 2020 Feb 24]. J Pediatric Infect Dis Soc. 2020;piaa014. doi:10.1093/jpids/piaa014

5. Marks KM, Kondo M, Dalai SC, et al. Diagnosis and Genotyping of Coxiella burnetii Endocarditis in a Patient with Prosthetic Pulmonary Valve Replacement Using Next-Generation Sequencing of Plasma Microbial Cell-Free DNA. Open Forum Infectious Diseases. 2019;6(6):1-4. doi:10.1093/ofid/ofz242

6. Shishido A, Vostal A, Mayer R, Ho Y, Baddley JW. Diagnosis of central nervous system invasive aspergillosis in a liver transplant recipient using microbial cell- free next generation DNA sequencing. 2021;(February):1-6. doi:10.1111/tid.13592

7. Eichenberger EM, de Vries CR, Ruffin F, et al. Microbial Cell-Free DNA Identifies Etiology of Bloodstream Infections, Persists Longer Than Conventional Blood Cultures, and Its Duration of Detection Is Associated With Metastatic Infection in Patients With Staphylococcus aureus and GramNegative Bacteremia. Clin Infect Dis. 2021;(Online ahead of print). doi:10.1093/cid/ciab742

8. Echeverria AP, Cohn IS, Danko DC, et al. Sequencing of Circulating Microbial Cell-Free DNA Can Identify Pathogens in Periprosthetic Joint Infections. Journal of Bone and Joint Surgery. 2021;103(18):1705-1712. doi:10.2106/jbjs.20.02229

9. Hogan CA, Yang S, Garner OB, et al. Clinical Impact of Metagenomic Next-Generation Sequencing of Plasma Cell-Free DNA for the Diagnosis of Infectious Diseases: A Multicenter Retrospective Cohort Study. Clinical Infectious Diseases. 2021;72(2):239-245. doi:10.1093/cid/ciaa035

10. Rossoff $\mathrm{J}$, Chaudhury $\mathrm{S}$, Soneji $\mathrm{M}$, et al. Noninvasive diagnosis of infection using plasma nextgeneration sequencing: A single-center experience. Open Forum Infectious Diseases. 2019;6(8). doi:10.1093/ofid/ofz327 
11. Benamu E, Gajurel K, Anderson JN, et al. Plasma Microbial Cell-free DNA Next Generation Sequencing in the Diagnosis and Management of Febrile Neutropenia. doi:10.1093/cid/ciab324/6236109

12. Noe M, Shishido AA, Kapil Saharia ; Luethy P. Clinical Utility and Impact of the Metagenomic Microbial Plasma Cell-Free DNA Next-Generation Sequencing Assay on Treatment Decision: A SingleCenter Retrospective Study. Open Forum Infectious Diseases. 2021;8(Supplement 1):S432-S433. https://academic.oup.com/ofid/article/8/Supplement_1/S432/6450531

\section{Supplementary Files}

This is a list of supplementary files associated with this preprint. Click to download.

- ClinicalUtilitymcfDNARAWDATAV2.xIsx 